\title{
A Rapid Serologic Diagnosis Sensor for Taenia Solium Cysticercosis
}

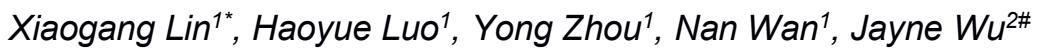 \\ ${ }^{1}$ Key Laboratory of Optoelectronic Technology and System of the Education Ministry of China, \\ Chongqing University, Chongqing 400044, China \\ ${ }^{2}$ Department of Electrical Engineering and Computer Science, the University of Tennessee, Knoxville, \\ TN 37996, USA
}

* corresponding author, xglin@cqu.edu.cn

\# co-corresponding author, jwu10@utk.edu

\begin{abstract}
:
Cysticercosis is a helminth zoonosis caused by Taenia solium, particularly in developing countries. Enzyme-linked Immunosorbent Assay (ELISA) and enzyme immunoelectrotransfer blot (EITB) are important methods for the detection of not only infected persons but also infected pigs. However, ELISA and EITB require testing in a laboratory facility and can be time consuming and labor intensive. In this study, we report a new, simple, rapid, highly sensitive and specific sensor based on an antigen probe $(\mathrm{rT} 24 \mathrm{H})$ and an AC electrokinetics (ACEK) capacitive sensing method. The impedance assay was developed by incorporating the ACEK effect seamlessly with the signal interrogation process for target macromolecule enrichment and binding process monitoring. As a result, binding time between the antigen-antibody is reduced from hours to 2 minutes. This method can simplify the complicated operation process of ELISA greatly, and transform the change of the immune response into the change of electrical parameters. In our tests, 82 blind samples were tested. Our method had a sensitivity of $95.31 \%$ and a specificity of $56 \%$. All of the serum samples were tested by the Centers for Disease Control and Prevention (CDC). Therefore, capacitive biosensing by microelectrodes holds great promise as a director, single-step assay procedure for point-of-care applications.
\end{abstract}

Key words: AC electrokinetics; Cysticercosis; Immunosensor; Capacitive sensing; Point of care diagnosis.

\section{Acknowledgements}

$X$. Lin acknowledges the support of the National Natural Science Foundation of China (Project No. 61377001). 